\title{
A NOTE ON ASYMPTOTIC STABILITY
}

\author{
OTTO PLAAT
}

1. Introduction. We consider a system of linear differential equations of the form

$$
\dot{x}=[A+\epsilon B(t)] x
$$

where $x$ is an $m$-vector, $A$ and $B(t)$ are complex $m \times m$ matrices, $A$ is constant and skew-Hermitian $\left(A^{*}=-A\right), B$ is continuous for all real $t$ and of period $\omega>0$, and $\epsilon$ is a small positive number. The problem of deciding the asymptotic behavior of the solutions of such a system is a common one in perturbation theory. The standard procedure for establishing the asymptotic stability of the trivial solution is to show that the characteristic multipliers have modulus less than 1 by expanding them in powers of $\epsilon[1]$. If $A$ and $B$ depend on a number of parameters, as is usually the case, the computations may be formidable. However, it is possible to exploit the fact that $A$ is skewHermitian to obtain a sufficient condition for asymptotic stability which considerably reduces the computational labor-in some cases to little more than the inspection of a certain Hermitian matrix.

We employ the following terminology. If $\phi(t), W(t)$ are $m \times m$ matrices continuous for real $t$ and $\dot{W}(t)=\phi(t) W(t), W(0)=I$, we say that $W$ is the fundamental matrix of $\dot{x}=\phi(t) x . C^{*}$ denotes the adjoint (conjugate transpose) of $C$.

Theorem. Let $A$ be skew-Hermitian and $B(t)$ continuous and of period $\omega>0$. Let $W_{0}$ be the fundamental matrix of $\dot{x}=A x$ and define $C_{n}=\int_{0}^{n \omega} W_{0}^{*}(t) B(t) W_{0}(t) d t$. If for some positive integer $n, C_{n}+C_{n}^{*}$ is negative definite, then for sufficiently small positive $\epsilon$ the trivial solution of (1) is asymptotically stable.

2. Lemmas. All matrices will be $m$-square. $\|T\|$ is the operator norm of $T$,

$$
\|T\|=\max \{\|T x\| ;\|x\|=1\}, \quad \text { where }\|x\|=\left[\sum_{i=1}^{m} x_{i} \bar{x}_{i}\right]^{1 / 2} .
$$

$\rho[T]$ is the maximum of the absolute values of the eigenvalues of $T$. Thus $\|T\|^{2}=\rho\left[T T^{*}\right]$. We note that if $W_{0}$ is the fundamental matrix of $\dot{x}=A x$, where $A^{*}=-A$, then $W_{0}^{*}(t)=W_{0}^{-1}(t)$. We choose to circumvent Floquet's theorem by means of the following trivial lemma.

Received by the editors June 9, 1967. 
LEMMA 1. Let $W$ be the fundamental matrix of $\dot{x}=\phi(t) x, \phi$ continuous and of period $\omega>0$. $W(t) \rightarrow 0$ as $t \rightarrow \infty$ if (and only if) $\|W(n \omega)\|<1$ for some positive integer $n$.

Proof. $W(s+n \omega)=W(s) W^{n}(\omega)$ for all real $s$ and integers $n$. Since any $t$ can be written $t=s+n \omega, 0 \leqq s<\omega$, we have $W(t)=W(s) W^{n}(\omega)$, where $0 \leqq s<\omega$. The condition of asymptotic stability of the trivial solution, $W(t) \rightarrow 0$ as $t \rightarrow \infty$, thus takes the form $W^{n}(\omega) \rightarrow 0$ as $n \rightarrow \infty$. But this is equivalent to $\left\|W^{n}(\omega)\right\|<1$ for some $n>0$. Since $W^{n}(\omega)$ $=W(n \omega)$, the lemma is proved.

Lemma 2. Let $V(\epsilon)=I+\epsilon C+\epsilon^{2} R(\epsilon)$, where $C$ is a matrix such that $C+C^{*}$ is negative definite, and $R(\epsilon)$ is continuous in $\epsilon$. Then $\|V(\epsilon)\|<1$ for sufficiently small positive $\epsilon$.

Proof. $\|V(\epsilon)\|^{2}=\rho\left[V(\epsilon) V^{*}(\epsilon)\right]$. Multiplying out yields $V(\epsilon) V^{*}(\epsilon)$ $=I+\epsilon T(\epsilon)$, where $T(\epsilon)=C+C^{*}+\epsilon S(\epsilon), S$ being continuous and Hermitian. By continuity, there is $\epsilon_{0}>0$ such that $T(\epsilon)$ is negative definite for $|\epsilon|<\epsilon_{0}$. It follows that if $0<\epsilon<\epsilon_{0}$ and $\lambda$ is an eigenvalue of $V(\epsilon) V^{*}(\epsilon)$, then $0 \leqq \lambda<1$.

3. Proof of the Theorem. Let $W(t, \epsilon)$ be the fundamental matrix of (1) and write $W(t, \epsilon)=W_{0}(t) V(t, \epsilon)$. Then

$$
V(t, \boldsymbol{\epsilon})=I+\epsilon \int_{0}^{\iota} W_{0}^{-1}(s) B(s) W_{0}(s) V(s, \boldsymbol{\epsilon}) d s .
$$

Substituting for $V$ in the integrand and replacing $W_{0}^{-1}$ by $W_{0}^{*}$ yields

$$
V(t, \epsilon)=I+\epsilon \int_{0}^{t} W_{0}^{*}(s) B(s) W_{0}(s) d s+\epsilon^{2} R(t, \epsilon),
$$

where $R$ is continuous. Let $n$ be a positive integer for which $C_{n}+C_{n}^{*}$ is negative definite. Then by Lemma $2,\|V(n \omega, \epsilon)\|<1$ for sufficiently small positive $\epsilon$. Since $W_{0}$ is unitary, $\|W(t, \epsilon)\|=\|V(t, \epsilon)\|$. Lemma 1 concludes the proof.

The theorem is easily extended to the case that $B=B(t, \epsilon)$ provided $B$ and $\partial B / \partial \epsilon$ are continuous for $|\epsilon|$ small. Except for the replacement of $B(t)$ by $B(t, 0)$ in the definition of $C_{n}$, the theorem and proof are unaltered.

The freedom offered by the arbitrariness of $n$ can result in an appreciable computational simplification. If, for example, the off-diagonal entries of $C_{n}+C_{n}^{*}$ are bounded in $n$, while the diagonal entries tend to $-\infty$ with increasing $n$, the matrix is obviously negative definite for large $n$. Examples suggest that this situation is not unusual. 
The author wishes to thank H. A. Antosiewicz for a suggestion which shortened the proof of the theorem.

\section{REFERENCE}

1. I. G. Malkin, Theory of stability of motion, translated from Russian, AEC-tr3352, U. S. Atomic Energy Commission, Oak Ridge, Tenn. p. 224. (Available from the Office of Technical Services, Department of Commerce, Washington, D. C.)

University of SAN Francisco 